\title{
The role of lipids in the pathogenesis and treatment of type 2 diabetes and associated co-morbidities
}

\author{
Derek M. Erion ${ }^{1}$, Hyun-Jun Park $^{2}$ \& Hui-Young Lee ${ }^{2,3, *}$ \\ ${ }^{1}$ Takeda Pharmaceuticals 350 Massachusetts Ave. Cambridge, MA, 02139, USA, ${ }^{2}$ Department of Molecular Medicine, ${ }^{3}$ Korea Mouse \\ Metabolic Phenotyping Center, Lee Gil Ya Cancer and Diabetes Institute, School of Medicine, Gachon University, Incheon 21999, Korea
}

In the past decade, the incidence of type 2 diabetes (T2D) has rapidly increased, along with the associated cardiovascular complications. Therefore, understanding the pathophysiology underlying T2D, the associated complications and the impact of therapeutics on the T2D development has critical importance for current and future therapeutics. The prevailing feature of T2D is hyperglycemia due to excessive hepatic glucose production, insulin resistance, and insufficient secretion of insulin by the pancreas. These contribute to increased fatty acid influx into the liver and muscle causing accumulation of lipid metabolites. These lipid metabolites cause dyslipidemia and non-alcoholic fatty liver disease, which ultimately contributes to the increased cardiovascular risk in T2D. Therefore, understanding the mechanisms of hepatic insulin resistance and the specific role of liver lipids is critical in selecting and designing the most effective therapeutics for T2D and the associated co-morbidities, including dyslipidemia and cardiovascular disease. Herein, we review the effects and molecular mechanisms of conventional anti-hyperglycemic and lipid-lowering drugs on glucose and lipid metabolism. [BMB Reports 2016; 49(3): 139-148]

\section{INTRODUCTION}

Intake of high-calorie food and sedentary lifestyle are factors contributing to the worldwide outbreak of metabolic diseases including obesity and type 2 diabetes (T2D) (1). An epidemic increase in metabolic diseases is forecasted to occur within the next decade (2). T2D and obesity are closely associated with one another. Both increase the risk of cardiovascular diseases (CVD), the leading cause of death in diabetic subjects (3). Since T2D is defined as hyperglycemia, the aim of diabetes

*Corresponding author. Tel: +82-32-899-6241; Fax: +82-32-8996039; E-mail: hylee@ gachon.ac.kr

http://dx.doi.org/10.5483/BMBRep.2016.49.3.268

Received 25 December 2015

Keywords: Cardiovascular disease, Diabetic dyslipidemia, Lipid metabolites, Insulin resistance, Type 2 diabetes management has been focused to reduce fasting blood glucose concentrations to normoglycemic range $(\sim 4$ to $6 \mathrm{mmol} / \mathrm{L})$. However, while the glucose-centric view of anti-diabetic treatments benefit microvascular complications associated with $\mathrm{T} 2 \mathrm{D}$, this view has limitations in fixing the underlying pathophysiology of the disease: insulin resistance (4).

Although, glucose is the key aspect of T2D, one often overlooked feature is the relationship between glucose control and its impact on lipids (5). Furthermore, lipids and glucose fluxes are closely interlinked through the intersection of metabolic pathways at acetyl-CoA formation. The perturbation of one pathway can indirectly affect another pathway. Given the association among lipids, insulin resistance, T2D and CVD, understanding the pathophysiology of T2D development from both glucose and lipid-view is critical to allow the discovery of the best targets for future therapeutics for T2D and its comorbidities.

In this review, we summarize the molecular mechanisms and the relationship between lipids and the pathogenesis of T2D and CVD. We also address the impact of T2D therapies on hepatic and circulating lipids. Last, we assess the impact of lipid therapies on glucose homeostasis.

\section{PATHOPHYSIOLOGY OF TYPE 2 DIABETES AND ASSOCIATED CVD}

Insulin resistance in the pathogenesis of T2D

Although the lack of insulin production and secretion by pancreatic $\beta$-cells are contributing factors to fasting hyperglycemia at later stages of $\mathrm{T} 2 \mathrm{D}$, insulin resistance is a factor that significantly contributes to the early development and progression into T2D (6). Insulin resistance is defined as diminished insulin signaling in normally insulin-responsive tissues such as liver, muscle, and adipose. The main traits of insulin resistance are decreased glycogen synthesis and glucose uptake in skeletal muscle $(7,8)$, defects in glycogen synthesis, and increased rates of gluconeogenesis in liver $(9,10)$. Skeletal muscle has been implicated as the predominant site of insulin-mediated glucose uptake during the postprandial stage in non-diabetic tissue (11). In contrast, during the fasting state, hepatic gluconeogenesis has been considered a main determinant for fasting plasma glucose concentrations and is de- 
termined, in part by plasma insulin concentrations (12). Insulin resistance in adipose tissue also has a significant contribution to hyperglycemia since adipose tissue is the major storage site of gluconeogenic substrates and energy, like fatty acids and glycerol. Thus, elevated lipolysis can increase hepatic gluconeogenesis (13). In addition, pathologically increased fatty acid influx into the liver induces aberrant increases in intracellular lipids. Accumulation of specific lipid metabolites contributes to lipid-induced hepatic insulin resistance. Increased intracellular lipids also leads to hyperglycemia, as well as diabetic dyslipidemia associated with increased CVD risk (14).

\section{Molecular mechanisms of lipid-induced insulin resistance} Mounting evidence has indicated that elevated intracellular lipid metabolite concentrations are associated with diminished insulin sensitivity in both liver and skeletal muscle. The specific molecular mechanism of lipid-induced insulin resistance has been thoroughly reviewed in previous papers (15-17). Excessive accumulation of toxic lipid metabolites, such as diacylglycerol (DAG) and ceramide species, provide a putative causal link between increased tissue lipids and hepatic insulin resistance via modulating insulin signaling $(16,17)$. DAG reduces insulin-stimulated phosphorylation of IRS2 and IRS2-associated $\mathrm{PI}(3) \mathrm{K}$ activity through protein kinase-C epsilon (PKCE) activation. Additional data demonstrates ceramide inhibits phosphorylation of Akt2 (17). Both DAG and ceramide result in the impaired action of insulin to activate glycogen synthesis and suppress gluconeogenesis. The crucial role of PKC $\varepsilon$ in mediating lipid-induced hepatic insulin resistance has been demonstrated by knocking down expression of PKC $\varepsilon$ in the liver using an antisense oligonucleotide in rodents (18). In human subjects, intrahepatic DAG content had the best correlation with hepatic insulin resistance compared to other lipid intermediates such as hepatic ceramide, acylcarnitines or inflammatory markers. However, these data do not necessarily show causation (19).

Alternatively, ceramide or other sphingolipids metabolites are also hypothesized to contribute to the pathogenesis of T2D and CVD, because they disrupt insulin sensitivity, pancreatic $\beta$ cell function and vascular reactivity (17). In a rodent model, hepatic ceramide content increased in rodents fed a high-fat diet (20). Reducing tissue ceramide content can, in certain cases, ameliorate insulin resistance and steatohepatitis $(17,21)$. Although, the causal role of ceramide remains to be tested using transgenic model, human studies demonstrate ceramide content is positively associated with metabolic diseases in various tissues such as muscle, plasma, and adipose tissue (22, 23). Elevated intracellular lipid metabolite concentrations have crucial roles in the pathogenesis of metabolic diseases including T2D, Non-alcoholic fatty liver disease (NAFLD), and CVD.

Type 2 diabetes and its co-morbidities including dyslipidemia, CVD and Nonalcoholic Steatohepatitis (NASH) T2D and its associated vascular complications are not independent diseases and are linked in part by dyslipidemia. Diabetic dyslipidemia resulting from lipotoxicity is a state characterized by changes in the lipid profile, including increases in triglycerides and in lipid metabolites such as DAG, ceramides, free fatty acids, low density lipoprotein (LDL) cholesterol, and low level of high density lipoprotein (HDL) cholesterol (24). Although the precise mechanisms for dyslipidemia in diabetes are not known, insulin resistance contributes to the underlying pathophysiological causes in addition to over-nutrition and obesity (14). In the liver, concomitant with impaired insulin action on glucose metabolism, lipogenesis remains unaffected (25). Therefore, unsuppressed hepatic glucose production along with increased lipogenesis likely worsens the development of hepatic steatosis and dyslipidemia (16). In other words, insulin resistance can cause both tissue and circulating lipid abnormalities, thereby partially linking diabetic dyslipidemia to increased CVD risk.

Dyslipidemia is a contributing mechanism by which insulin resistance increases CVD risk in T2D individuals (26). Excessive production of very low density lipoproteins (VLDLs) in the liver and increased adipocyte free fatty acid flux from lipolysis under insulin resistant conditions also result in tissue and circulating lipid abnormalities. Since insulin is critical in the inhibition of hepatic VLDL formation in the liver and lipolysis in adipose tissue $(14,27)$, recovery of insulin action in these tissues is one of the key strategies to deal with abnormal lipid profiles found in T2D patients.

Hepatic steatosis, defined as excessive hepatic fat deposition, is a common co-morbidity of T2D and obesity (28). Although often considered a benign condition that can be stable over decades, the accumulation of hepatic fat is thought to be a pre-requisite for the development of NASH and the development of hepatic fibrosis (29). Additionally, studies suggest NAFLD is an independent risk factor for CVD (30). $\mathrm{NASH}$ was reported in $\sim 12 \%$ of the United States population, while $\sim 46 \%$ of the Unites States population was found to have NAFLD (31). T2D is thought to accelerate the progression of $\mathrm{NASH}$ and fibrosis through the effects of hyperglycemia, inflammation, and lipid overload. Therefore, targeting tissue lipid has the potential to treat T2D and its co-morbidities.

\section{THE EFFECT OF CURRENT TYPE 2 DIABETES THERAPIES ON LIPIDS}

\section{Metformin}

Metformin is currently the standard of care for glycemic control in diabetic patients (32). Metformin alleviates hyperglycemia via suppression of hepatic gluconeogenesis and enhancement of tissue glucose uptake $(33,34)$. In terms of glucose and lipid homeostasis, pre-clinical studies show that metformin increases AMP-activated kinase (AMPK) phosphorylation and downstream responses of glucose and hepatic lipid regulation, including cholesterol and triglycerides (35, 36). However, clinical studies investigating the use of metformin have shown 
minimal benefits on LDL cholesterol and triglycerides, when correcting for glucose control in diabetic patients (37). Furthermore, in patients with defined NASH, no histological changes were observed following a 52-week NASH trial in non-diabetics with insulin resistance (38).

The extent of AMPK activation and reduction of lipids by metformin in pre-clinical trials may be due to the use of supra-pharmacological doses resulting in metformin circulating concentrations greater than $1 \mathrm{mM}$ (39). Although, many studies have demonstrated some clinical benefits on NAFLD and reductions in circulating liver enzymes, metformin mechanistically and clinically appears to have limited benefits in reversing the pathophysiology of NASH patients. The lack of robust translatability of the impact of metformin on lipids from the pre-clinical mechanistic work to the clinical setting may be due to the supra-pharmacological doses used in the pre-clinical trials. Multiple studies have shown the glycemic effect of metformin occurs independently of AMPK activation. Recently, the specific molecular mechanism of metformin inhibition of hepatic gluconeogenesis has been proposed to occur by non-competitive inhibition of mitochondrial glycerophosphate dehydrogenase 2 through effects on the redox state (39). The impact of this redox cycle inhibition on lipids is still under analysis.

\section{Thiazolidinediones (TZDs)}

TZDs were initially discovered at Takeda as molecules to improve metabolism and have been subsequently described in publications since 1983 (40). TZDs exert their metabolic benefits as agonists of the nuclear hormone receptor, peroxisome proliferator-activated receptor gamma (PPAR-gamma), which controls a variety of key metabolic processes such as insulin sensitivity, adipogenesis, and lipid metabolism (41). TZDs induce sustained and well controlled glycemic balance through insulin sensitization. PPAR-gamma is primarily expressed in white adipose tissue. Activation of PPAR-gamma causes adipogenesis, increases in mitochondrial respiration, and browning of adipose tissue $(42,43)$. These molecular mechanisms resulted in controlling effects on plasma glycemia and a more durable glycemic control when compared to metformin and sulfonylureas (44).

Regarding the lipid control, TZDs have substantial benefits on hepatic lipids as demonstrated in numerous clinical studies. In a trial in subjects with T2D and defined NASH, pioglitazone treatment was found to normalize circulating plasma ALT and AST concentrations, as well as, reduce hepatic fat concentrations by $54 \%$ (45). Subsequently, the PIVENS trial investigated the use of Vitamin E or pioglitazone for the treatment of $\mathrm{NASH}$ in non-diabetics. Although the primary endpoint, after 52 weeks in subjects treated with pioglitazone, did not reach statistical significance for histological improvements in NASH (46), secondary endpoint scores were met, as in reduction in steatosis, lobular inflammation, and reduction in the non-alcoholic fatty liver disease. Furthermore, in the FLIRT trial, which evaluated rosiglitazone for the treatment of $\mathrm{NASH}$, found an improvement in steatosis. However, there were no additional benefits on other histological endpoints (47).

The molecular mechanisms behind the reduction of hepatic triglycerides and improvements in NASH observed with subjects treated with TZDs are evolving. One hypothesis is the "lipid steal" model in which TZDs cause adipose tissue expansion through the formation of mature adipocytes from adipose tissue progenitor cells and the browning of mature adipocytes through the stabilization of PRDM16. In another study, TZDs are shown to function by sequestering lipids in adipose tissue rather than in the liver (48).

According to results from Reitman and colleagues, white adipose tissue is the most important site for lipid sequestration under TZD treatment (42). A-ZIP/F-1 mice, which do not have adipose tissue, exhibit lipid accumulation in the liver similar to lipodystrophic diabetes, while implantation of adipose tissue to A-ZIP/F-1 mice improve hepatic steatosis (42). Consequently, TZD treatment induces hepatic lipid reduction related to the possibility of improvements in insulin resistance. A second hypothesis for reduction of hepatic steatosis by TZD is through the activation of PPAR-gamma in white adipocytes, which allows the dysfunctional adipose tissue found in diabetes and the metabolic syndrome, to be returned to functional adipose tissue for the storage of lipids. This in turn reduces the circulating free fatty acids and the fat content in muscle and liver tissue.

An alternative hypothesis is through the off target inhibition of MCP1 and MCP2, the mitochondrial pyruvate carrier transporter, by TZDs. Specific deletion of MPC in the liver has led to pyruvate-alanine cycling and reductions in hepatic gluconeogenesis (49). Development of specific MCP inhibitors is currently in clinical trials for T2D and NASH (50). Signs of PPAR-gamma activation, water retention, and increases in circulating adiponectin were also observed in some of these clinical studies (50). MCP inhibitors devoid of PPAR-gamma activity will be needed to determine the specific impact of MCP inhibition on lipids and glucose.

Although, a potent insulin sensitizer, prescriptions of TZDs have decreased due to safety concerns, such as bone fractures and potential cancers, in addition to the less desirable effects on edema and weight gain (41).

\section{Sulfonylurea (SU)/Insulin}

Sulfonylurea (SU) is an insulin secretagogue, which binds to the ATP-sensitive $\mathrm{K}^{+}$channel and facilitates insulin secretion. The use of $\mathrm{SU}$ is associated with robust reductions in $\mathrm{HbA1c}$; however, the durability of therapeutic effect by SU treatment has been questioned (51). By stimulating insulin secretion, SU treatment exhibits improvement in glycemic control, including suppression of hepatic gluconeogenesis, which results in reduced fasting blood glucose concentrations. However the use of SU agents is associated with increased hypoglycemia risk (52).

In terms of effects on lipids, it was reported that SU agents have the ability to reverse the abnormal lipid composition of 
diabetics including the high triglyceride:ApoB ratio (53). During $\mathrm{SU}$ therapy, triglyceride production and the triglyceride:ApoB ratio were reduced and VLDL clearance rate improved (53). In an additional study, total cholesterol and LDL cholesterol were markedly reduced with an increase in the HDL:LDL cholesterol ratio (54). However, larger studies failed to show significant effects on triglycerides in patients treated with SU (55).

Basal insulin, such as glargine, has been a mainstay in type 1 and 2 diabetes since the early 2000s. In meta-analyses, basal insulin exhibited modest reductions in LDL cholesterol and triglycerides. However, there was no cardiovascular benefit in subjects treated with basal insulin in the Outcome Reduction With Initial Glargine Intervention (ORGIN) trial (56).

Glargine was shown to reduce the total liver fat index in a 12 -week study in patients with poorly controlled T2D. The mechanism behind the effects of SUs and insulin on lipids is presumably through the inhibitory effect of insulin on adipose tissue lipolysis and increased clearance of TG-rich chylomicrons through the activation of lipoprotein lipase (LPL).

\section{Glucagon-like peptide-1 (GLP-1) and Dipeptidyl peptidase-IV (DPP-IV) inhibitors}

Gut derived glucagon-like peptide-1 (GLP-1) is an insulin secretion stimulator that promotes insulin action. Although the direct effect of GLP-1 on human hepatocytes is controversial, GLP-1 treatment improves hepatic steatosis in diet-induced obesity (DIO) and in genetically obese models. In human, GLP-1 treatment caused significant reductions in hepatic triglycerides in longer clinical studies (57-59). The key mode of action of GLP-1 is an increase of cyclic AMP (cAMP) levels in hepatocytes, which leads to downstream activation of AMPK in the liver. Activation of AMPK directly regulates transcription of SREBP-1c and in turn, lipogenic downstream genes, such as acetyl-CoA carboxylase, fatty acid synthase, and stearoyl-CoA desaturase-1 are down-regulated; although the detection of the GLP-1 receptor on human hepatocytes has been controversial.

Therefore, in pre-clinical studies, steatosis is resolved with direct activation of GLP-1 receptor. Additionally to NAFLD, liraglutaide, a long acting peptide GLP-1 agonist, was shown to be safe and efficacious in patients with non-alcoholic steatohepatitis in the LEAN trial. In this trial, $39 \%$ of overweight patients with clinical signs of NASH treated with $1.8 \mathrm{mg}$ of liraglutade resolved their NASH compared to only $9 \%$ in the placebo group (60). Liraglutide has also been shown to positively influence circulating triglycerides (61).

Dipeptidyl peptidase-IV (DPP-IV) inhibitors are oral hypoglycemic, which inhibit a deactivation of glucagon-like peptide-1 (GLP-1). Due to its close relationship with GLP-1, the DPP-IV inhibitor mechanism of action was proposed to be by increasing insulin secretion. However, precise mode of action of DPP-IV inhibitors remains unclear. Recently, it has been demonstrated that GLP-1 or DPP-IV treatment causes AMPK activation through an increase in CAMP (57). Since AMPK phosphorylation directly prevents hepatic lipogenesis, this pro- vides a clear explanation of the results from pre-clinical and clinical studies.

Aroor et al. showed that DPP-IV inhibitor treatment in the $\mathrm{DIO}$ model rescued insulin resistance and significantly reduced triglycerides, DAG, and certain fatty acid species content (62). Furthermore, hepatic triglyceride secretion was reversed by a DPP-IV inhibitor (62). Pre-clinical data is also consistent with results from human clinical studies, concerning hepatic lipid content $(63,64)$. DPP-IV agents have not shown any cardiovascular benefit and some DPP-IV inhibitors are associated with increased hospitalization due to heart failure (65). Although GLP-1 and DPP-IV both show substantial positive effects on hepatic lipids, its cardiovascular benefit has yet to be demonstrated.

\section{Sodium/Glucose co-transporter 2 (SGLT2) inhibitors}

SGLT2 inhibitors are a new class of anti-diabetic drugs which lower blood glucose by inhibition of glucose reabsorption in the renal proximal tubule through SGLT2 (66). Although SGLT2 inhibitor treatment brings about reduction of insulin secretion and increased endogenous glucose production as a counter-regulatory response to therapeutic glycosuria, this therapy improves pancreatic $\beta$-cell function, insulin action (66), and shifts the substrate utilization to lipids in the case of chronic treatment $(67,68)$.

Yokono et al. investigated the effects of ipragliflozin, a SGLT2 inhibitor, on lipid metabolism in rats fed high fat diets (68). Under treatment, visceral and subcutaneous fat masses decreased and lipid utilization concomitant with effects on lipolysis and fatty acid oxidation improved (68). In the clinical setting, SGLT2 inhibition was shown to cause a modest reduction in body weight and blood pressure, although there were increases in circulating LDL concentrations $(69,70)$. Recently, patients administered empagliflozin at either $10 \mathrm{mg}$ or $25 \mathrm{mg}$ showed improvement in cardiovascular outcomes and death from any cause (71). The molecular mechanisms behind the improvement in cardiovascular outcomes are still being elucidated.

\section{Glucokinase activators and glucagon antagonists}

Both hepato-selective and systemic glucokinase activators have been developed for T2D along with glucagon antagonists in an attempt to rebalance the insulin:glucagon ratio/signaling and to reduce hepatic glucose output $(72,73)$. Glucokinase activation in the $\beta$-cell increases insulin secretion, however, is associated with lack of durable efficacy and inappropriate insulin secretion resulting in hypoglycemia. To circumvent these issues, hepato-selective glucokinase activators were developed to have glycemic control. Unfortunately, they were associated with increased circulating plasma triglycerides (74).

Studies from pre-clinical models showed that increased plasma triglycerides were likely due to increased secretion due to activation of the lipogenic transcription factor, carbohydrateresponsive element-binding protein (74). Pre-clinical studies 
using this compound made by Pfizer, predicted minimal changes in hepatic steatosis based on data from diabetic rats despite the elevated circulated triglycerides (74). Lastly, a glucagon antagonist developed by Merck has been associated with increased LDL cholesterol and attributed to increased cholesterol absorption, as demonstrated in pre-clinical studies (75). The reduction in hepatic glucose output via these pathways show robust changes in glycemia, however, the impact on lipids should be of careful consideration.

\section{THE EFFECT OF CIRCULATING LIPID-LOWERING DRUGS ON GLUCOSE AND LIPIDS}

\section{Niacin}

Niacin, also known as nicotinic acid or vitamin $\mathrm{B}_{3}$ has the ability to modify lipid abnormalities, by lowering LDL cholesterol and triglyceride while increasing HDL cholesterol (76). Niacin has been prescribed to patients with a high risk of CVD, such as atherosclerosis. Alternation of abnormal lipid species is mediated by indirect inhibition against VLDL formation (77). In particular, niacin down-regulates apolipoprotein B-100 production (78) and facilitates VLDL catabolism by activating LPL (79).

The effect of niacin in T2D has been controversial. With respect to glucose regulation, long-term treatment of nicotinic acid to treat CVD tends to increase the risk of T2D, because patients with impaired fasting glucose have shown elevated fasting blood glucose concentrations than normoglycemic groups (76). However, data from long-term clinical studies by Elam et al. report stable glucose homeostasis; although a slight increase in fasting blood glucose at the early stage of niacin treatment was observed (80). In conclusion, niacin has a positive effect on lipid regulation but does not have noticeable glucose lowering effect. The majority of clinical results do not mention any decrease in $\mathrm{HbA} 1 \mathrm{c}$ and fasting blood glucose $(76,80)$.

\section{Statins}

Statins are prominent cholesterol lowering drugs, which inhibit HMG-CoA reductase, an enzyme that participates in the key step of cholesterol synthesis. The LDL cholesterol lowering effects of statins and cardiovascular benefits have been reported in many studies $(81,82)$. In addition, other potential benefits including effects on glucose metabolism have been suggested (83). Ogawa and colleagues demonstrated beneficial effects of pravastatin against impaired glucose tolerance (84). In particular, pravastatin treatment was shown to increase plasma adiponectin levels and significant association between elevated level of adiponectin and a decrease in blood glucose levels at the $2 \mathrm{hr}$-post oral glucose tolerance test (OGTT) was observed (84). Despite these studies, conflicting results from other randomized statin trials implicated a slight increase in the risk of new-onset diabetes $(85,86)$. In conclusion, statin therapy against T2D has no positive effect, even though statin therapy markedly reduced lipid species, including LDL cholesterol.

\section{Proprotein convertase subtilisin/kexin type 9 (PCSK9) inhibitor}

PCSK9 inhibitor blocks the binding of PCSK9 to the LDL cholesterol receptor and induces lysosomal degradation. As a result, more LDL cholesterol receptors remain on the cellular membrane and become available to remove LDL cholesterol from the blood. Alirocumab, a PCSK9 inhibitor under development, has entered a phase III clinical trial (87) and exhibits significantly more effective LDL cholesterol lowering, compared to ezetimibe (88). However, there were no considerable changes in HbA1c or fasting blood glucose (88), which could translate to limited efficacy on controlling glucose concentrations by PCSK9 inhibitors.

\section{Cholesteryl ester transfer protein (CETP) inhibitors}

CETP mediates the transportation of the cholesteryl ester group of $\mathrm{HDL}$ cholesterol to VLDLs. This exchange induces a decrease in HDL cholesterol and increases VLDLs and LDL cholesterol, the main culprits of atherosclerosis (89). Based on the mechanism of CETP, synthetic inhibitors effectively block transfer of cholesteryl ester from HDL cholesterol to improve the lipid profile. Almost all clinical trials delineate similar results showing that CETP inhibitor monotherapy or combined therapies with statins are an effective means to raise anti-atherogenic $\mathrm{HDL}$ cholesterol $(90,91)$. In terms of T2D, further research is necessary. However, some studies, such as Barter et al., show positive effects of long-term treatment of torcetrapib and statins on glucose metabolism, resulting in decreased blood glucose, insulin, and $\mathrm{HbA} 1 \mathrm{c}$, as well as, cholesterol profile improvement (92).

\section{THE EFFECT OF NOVEL METABOLIC DISEASE AGENTS ON LIPID AND GLUCOSE}

\section{Fibroblast growth factor 21 (FGF21)}

FGF21 is a metabolic hormone with several beneficial effects on glucose and lipid metabolism and insulin sensitivity in pre-clinical trials. Most pre-clinical, in vivo results support the anti-diabetic effects of FGF21, demonstrating both lipid and obesity-induced hyperglycemia reductions (93). The key mechanism of action of FGF21 is through the adiponectin coupled metabolic response. Lin et al. demonstrated the role of adiponectin as an effector of FGF21 by using adiponectin knockout mice (94). Additionally, Bernardo et al. showed that functioning brown adipose tissue was not necessary for the favorable metabolic effects of FGF21 supporting the importance of white adipose tissue (95). Furthermore, translational clinical research using an FGF21 analogue treatment resulted in marked improvements of lipid abnormalities, including decreases in LDL cholesterol and triglycerides. Metabolically beneficial effects were also confirmed in terms of body weight and fasting insulin levels with a slight decrease in glucose (96). Positive data from pre-clinical and translational research indicates the potential of FGF21 as a new class of drug for metabolic 
disorders, specifically in controlling dyslipidemia.

\section{Apolipoprotein C3 (APOC3) inhibitors}

APOC3 is one of the protein components of VLDLs and inhibits LPL (97). APOC3 inhibits hepatic uptake of triglyceride-rich lipoproteins by blocking the binding between apoB lipoproteins and their receptors (14). Genetic variants in APOC3 were discovered in Asian Indian men with high occurrence of NAFLD and insulin resistance (97). In Asian Indian individuals, as well as those of other ethnic groups, variants (C-482T, $\mathrm{T}-455 \mathrm{C}$ or both) in the insulin responsive element of the APOC3 gene, which can inhibit LPL and hepatic lipase, are associated with hypertriglyceridemia and hepatic steatosis (97). These polymorphisms are a gain of function mutation and lead to $\sim 30 \%$ higher plasma APOC3 concentrations and post-prandial hypertriglyceridemia (97). This accounts for the high occurrence of NAFLD and insulin resistance in this ethnic group (98). The effect of increased plasma APOC3 concentrations on the development of NAFLD and hepatic insulin resistance has been genetically validated in transgenic mice that have increased hepatic overexpression of human APOC3 (99). These mice are more prone to diet-induced NAFLD and DAG-PKCE-induced hepatic insulin resistance (99). The development of hepatic lipid accumulation was due to decreased hepatic VLDL secretion by postprandial hyperinsulinemia. Interestingly, the insulin suppression of hepatic gluconeogenesis was diminished despite of hyperinsulinemia (99), indicating hepatic insulin resistance occurred selectively by the suppression of gluconeogenesis rather than VLDL suppression. Moreover, the loss-of-function mutation in APOC3 has been linked to favorable lipid profiles and lower incidences of coronary artery disease (100-102).
More recently, a clinical trial showed remarkable improvements in familial chylomicronemia with inhibitors of APOC3 mRNA (103), suggesting a therapeutic potential of ApoC3 targeting against lipid-induced insulin resistance, NAFLD, dyslipidemia and CVD.

\section{Monoacylglycerol/Diacylglycerol acyltransferase inhibitors} Diacylglycerol acyltransferase (DGAT) is an enzyme that catalyzes triglyceride synthesis from DAG. There are two key isoforms, DGAT1 and DGAT2. DGAT1 is critical in intestinal lipid absorption. DGAT2 appears to be critical for the secretion of VLDL triglycerides. Inhibition of DGAT1 is associated with adverse gastrointestinal side effects that limit the clinical utility of DGAT1 inhibitors. Novartis has tested the potential of using DGAT1 inhibitors in NASH and has observed reductions in hepatic fat content (AASLD poster \#2147, http://www.aasld. org/events-professional-development/liver-meeting/scientificprogram/poster4).

Several in vivo studies have demonstrated the importance of DGAT2 on hepatic lipid handling. Knocking down DGAT2 using an anti-sense oligonucleotide resulted in reduction of hepatic steatosis (104-106). In preclinical trials, DGAT2 inhibition has been shown to increase insulin responsiveness, as assessed by the hyperinsulinemiceuglycemic clamp technique (107). Subsequently, efficacy of DGAT1 inhibitors for human applications was tested and results showed noticeable regulation of lipid abnormalities (108, 109).

Similarly, monoacylglycerol acyltransferase (MGAT) enzymes, which convert monoacylglycerol to DAG, a key mediator of insulin resistance, have recently been considered of potential relevance to the mechanisms of obesity-related hepatic

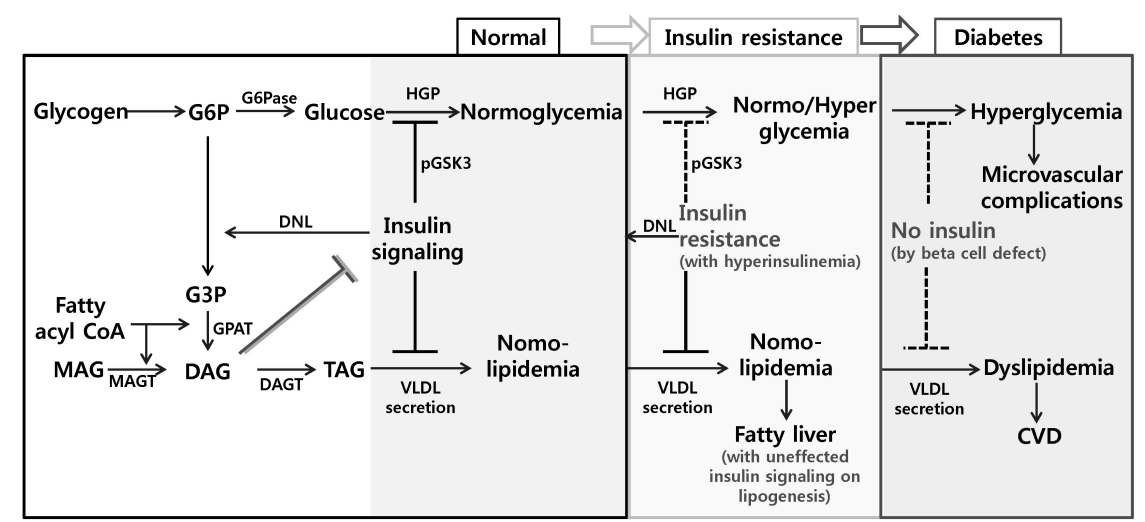

Fig. 1. A pathophysiological development of diabetes and associated co-morbidities. Concomitant with impaired insulin action on glucose metabolism and lipogenesis remains unaffected in the liver (25). Therefore, unsuppressed hepatic glucose production along with increased lipogenesis likely worsens the development of hepatic steatosis and dyslipidemia (16). This may partially explain why conventional anti-hyperglycemic agents have not shown benefits on the life-threatening macrovascular complications, including CVD atherosclerosis and coronary heart diseases. On the contrary, tissue lipid lowering diabetic drugs could have the potential to decrease both tissue and circulating lipid abnormalities, thereby partially linking diabetic dyslipidemia to increased CVD risk. HGP: hepatic glucose production, G6P: glucose-6-phosphate, DNL: de novo lipogenesis, DAG: diacylglycerol, TAG: triglyceride, VLDL: very low-density lipoprotein, MGAT: monoacylglycerol acyltransferase, DGAT: diglyceride acyltransferase. 
steatosis (110-112). In the fatty liver of rodents $(110,111)$ and humans (112), MGAT gene expression increases. However, weight loss in obese subjects is associated with improved insulin sensitivity and hepatic steatosis decreases (112). In diet-induced obese mice, inhibition of MGAT acts to cause a degree of lipid-induced hepatic insulin resistance, which can lead to hyperglycemia, as well as, diabetic dyslipidemia that is closely linked to CVD (Fig. 1). Thus, anti-diabetic drugs, which modulate tissue lipids, may be considered as a strategy to treat the underlying NAFLD, hepatic insulin resistance, and modify CVD risk.

\section{CONCLUSION}

In metabolism, glucose and lipids are highly interrelated. Affecting lipid or glucose metabolism can cause the flux of carbon to divert into an alternate pathway. Careful attention to the impact of pharmacological agents for T2D on lipids and conversely the impact of agents to control lipids on glycemic control should be made. Since metabolic diseases often include both adverse changes in lipids and glucose, future research should be directed to therapeutic strategies that treat the underlying pathophysiology of metabolic diseases to reduce both glucose and lipid concentrations.

\section{ACKNOWLEDGEMENTS}

We thank Thanh Erion, PhD for the careful input and review of this manuscript. Due to space limitation, this review cites only a limited number of studies that have been performed on the pharmacological agents described and does not contain a full list of citations to other studies that have been performed on these pharmacological agents. This work was supported by the Bio \& Medical Technology Development Program of the National Research Foundation (NRF) funded by the Ministry of Science, ICT \& Future Planning (NRF-2014R1A1A1002009 and NRF-2014M3A9D5A01073886).

\section{REFERENCES}

1. Zimmet P, Alberti KG and Shaw J (2001) Global and societal implications of the diabetes epidemic. Nature 414, 782-787

2. Guariguata L, Whiting DR, Hambleton I, Beagley J, Linnenkamp U and Shaw JE (2014) Global estimates of diabetes prevalence for 2013 and projections for 2035 . Diabetes Res Clin Pract 103, 137-149

3. Roger VL, Go AS, Lloyd-Jones DM et al (2012) Heart disease and stroke statistics-2012 update: a report from the American Heart Association. Circulation 125, e2-e220

4. UK Prospective Diabetes Study (UKPDS) Group (1998) Intensive blood-glucose control with sulphonylureas or insulin compared with conventional treatment and risk of complications in patients with type 2 diabetes (UKPDS 33). Lancet 352, 837-853
5. Bonora E, Formentini G, Calcaterra F et al (2002) HOMAestimated insulin resistance is an independent predictor of cardiovascular disease in type 2 diabetic subjects: prospective data from the Verona Diabetes Complications Study. Diabetes Care 25, 1135-1141

6. Wajchenberg BL (2007) beta-cell failure in diabetes and preservation by clinical treatment. Endocr Rev 28, 187-218

7. Rothman DL, Shulman RG and Shulman GI (1992) 31P nuclear magnetic resonance measurements of muscle glucose-6-phosphate. Evidence for reduced insulin-dependent muscle glucose transport or phosphorylation activity in non-insulin-dependent diabetes mellitus. J Clin Invest 89, 1069-1075

8. Shulman Gl, Rothman DL, Jue T, Stein P, DeFronzo RA and Shulman RG (1990) Quantitation of muscle glycogen synthesis in normal subjects and subjects with non-insulin-dependent diabetes by $13 \mathrm{C}$ nuclear magnetic resonance spectroscopy. N Engl J Med 322, 223-228

9. Hwang JH, Perseghin G, Rothman DL et al (1995) Impaired net hepatic glycogen synthesis in insulin-dependent diabetic subjects during mixed meal ingestion. A $13 \mathrm{C}$ nuclear magnetic resonance spectroscopy study. J Clin Invest 95, 783-787

10. Landau BR, Wahren J, Chandramouli V, Schumann WC, Ekberg K and Kalhan SC (1996) Contributions of gluconeogenesis to glucose production in the fasted state. J Clin Invest 98, 378-385

11. DeFronzo RA and Tripathy D (2009) Tripathy, Skeletal muscle insulin resistance is the primary defect in type 2 diabetes. Diabetes Care 32, S157-S163

12. DeFronzo RA, Ferrannini E and Simonson DC (1989) Simonson, Fasting hyperglycemia in non-insulin-dependent diabetes mellitus: contributions of excessive hepatic glucose production and impaired tissue glucose uptake. Metabolism 38, 387-395

13. Perry RJ, Camporez JP, Kursawe R et al (2015) Hepatic acetyl CoA links adipose tissue inflammation to hepatic insulin resistance and type 2 diabetes. Cell, 160, 745-758

14. Wu L and Parhofer KG (2014) Diabetic dyslipidemia. Metabolism 63, 1469-1479

15. Reaven GM (1988) Banting lecture 1988. Role of insulin resistance in human disease. Diabetes 37, 1595-1607

16. Samuel VT and Shulman Gl (2012) Mechanisms for insulin resistance: common threads and missing links. Cell $148,852-871$

17. Chaurasia B and Summers SA (2015) Ceramides Lipotoxic Inducers of Metabolic Disorders. Trends Endocrinol Metab 26, 538-550

18. Samuel VT, Liu ZX, Qu X et al (2004) Mechanism of hepatic insulin resistance in non-alcoholic fatty liver disease. J Biol Chem 279, 32345-32353

19. Magkos F, Su X, Bradley D et al (2012) Intrahepatic diacylglycerol content is associated with hepatic insulin resistance in obese subjects. Gastroenterology 142, 14441446 e2

20. Jurczak MJ, Lee AH, Jornayvaz FR et al (2012) Dissociation of inositol-requiring enzyme (IRE1alpha)-mediated c-Jun $\mathrm{N}$-terminal kinase activation from hepatic insulin 
resistance in conditional X-box-binding protein-1 (XBP1) knock-out mice. J Biol Chem 287, 2558-2567

21. Lee SY, Hong IK, Kim BR et al (2015) Activation of sphingosine kinase 2 by endoplasmic reticulum stress ameliorates hepatic steatosis and insulin resistance in mice. Hepatology 62, 135-146

22. Haus JM, Kashyap SR, Kasumov T et al (2009) Plasma ceramides are elevated in obese subjects with type 2 diabetes and correlate with the severity of insulin resistance. Diabetes 58, 337-343

23. Adams JM 2nd, Pratipanawatr T, Berria R et al (2004) Ceramide content is increased in skeletal muscle from obese insulin-resistant humans. Diabetes 53, 25-31

24. Mooradian AD (2009) Dyslipidemia in type 2 diabetes mellitus. Nat Clin Pract Endocrinol Metab 5, 150-159

25. Semple RK, Sleigh A, Murgatroyd PR et al (2009) Postreceptor insulin resistance contributes to human dyslipidemia and hepatic steatosis. J Clin Invest 119, 315-322

26. Rader DJ (2007) Effect of insulin resistance, dyslipidemia, and intra-abdominal adiposity on the development of cardiovascular disease and diabetes mellitus. Am J Med 120, S12-S18

27. Adiels M, Olofsson SO, Taskinen MR and Borén J (2008) Overproduction of very low-density lipoproteins is the hallmark of the dyslipidemia in the metabolic syndrome. Arterioscler Thromb Vasc Biol 28, 1225-1236

28. Bang KB and Cho YK (2015) Comorbidities and Metabolic Derangement of NAFLD. J Lifestyle Med 5, 7-13

29. Koo SH (2013) Nonalcoholic fatty liver disease: molecular mechanisms for the hepatic steatosis. Clin Mol Hepatol 19, 210-215

30. Athyros VG, Tziomalos K, Katsiki N, Doumas M, Karagiannis A and Mikhailidis DP (2015) Cardiovascular risk across the histological spectrum and the clinical manifestations of non-alcoholic fatty liver disease: An update. World J Gastroenterol 21, 6820-6834

31. Williams CD, Stengel J, Asike Ml et al (2011) Prevalence of nonalcoholic fatty liver disease and nonalcoholic steatohepatitis among a largely middle-aged population utilizing ultrasound and liver biopsy: a prospective study. Gastroenterology 140, 124-131

32. American Diabetes Association (2009) Standards of medical care in diabetes-2009. Diabetes Care 32, S13-S61

33. Klip A and Leiter LA (1990) Cellular mechanism of action of metformin. Diabetes Care 13, 696-704

34. Stumvoll M, Nurjhan N, Perriello G, Dailey G and Gerich JE (1995) Metabolic effects of metformin in non-insulin-dependent diabetes mellitus. $\mathrm{N}$ Engl J Med 333, 550-554

35. Kim YD, Park KG, Lee YS et al (2008) Metformin inhibits hepatic gluconeogenesis through AMP-activated protein kinase-dependent regulation of the orphan nuclear receptor SHP. Diabetes 57, 306-314

36. Zhou G, Myers R, Li Y et al (2001) Role of AMP-activated protein kinase in mechanism of metformin action. J Clin Invest 108, 1167-1174

37. Wulffelé MG, Kooy A, de Zeeuw D, Stehouwer CD and Gansevoort RT (2004) The effect of metformin on blood pressure, plasma cholesterol and triglycerides in type 2 diabetes mellitus: a systematic review. J Intern Med 256,
1-14

38. Shields WW, Thompson KE, Grice GA, Harrison SA and Coyle WJ (2009) The Effect of Metformin and Standard Therapy versus Standard Therapy alone in Nondiabetic Patients with Insulin Resistance and Nonalcoholic Steatohepatitis (NASH): A Pilot Trial. Therap Adv Gastroenterol 2, 157-163

39. Madiraju AK, Erion DM, Rahimi Y et al (2014) Metformin suppresses gluconeogenesis by inhibiting mitochondrial glycerophosphate dehydrogenase. Nature 510, 542546

40. Fujita T, Sugiyama Y, Taketomi S et al (1983) Reduction of insulin resistance in obese and/or diabetic animals by 5-[4-(1-methylcycloheylmethoxy)benzyl]-thiazolidine2,4-dione (ADD-3878, U-63,287, ciglitazone), a new antidiabetic agent. Diabetes 32, 804-810

41. Soccio RE, Chen ER and Lazar MA (2014) Thiazolidinediones and the promise of insulin sensitization in type 2 diabetes. Cell Metab 20, 573-591

42. Chao L, Marcus-Samuels B, Mason MM et al (2000) Adipose tissue is required for the antidiabetic, but not for the hypolipidemic, effect of thiazolidinediones. J Clin Invest 106, 1221-1228

43. Ohno H, Shinoda K, Spiegelman BM and Kajimura $S$ (2012) PPARgamma agonists induce a white-to-brown fat conversion through stabilization of PRDM16 protein. Cell Metab 15, 395-404

44. Kahn SE, Haffner SM, Heise MA et al (2006) Glycemic durability of rosiglitazone, metformin, or glyburide monotherapy. N Engl J Med 355, 2427-2443

45. Belfort R, Harrison SA, Brown K et al (2006) A placebo-controlled trial of pioglitazone in subjects with nonalcoholic steatohepatitis. N Engl J Med 355, 2297-2307

46. Sanyal AJ, Chalasani N, Kowdley KV et al (2010) Pioglitazone, vitamin $\mathrm{E}$, or placebo for nonalcoholic steatohepatitis. N Engl J Med 362, 1675-1685

47. Ratziu V, Giral P, Jacqueminet S et al (2008) Rosiglitazone for nonalcoholic steatohepatitis: one-year results of the randomized placebo-controlled Fatty Liver Improvement with Rosiglitazone Therapy (FLIRT) Trial. Gastroenterology $135,100-110$

48. Cariou B, Charbonnel B and Staels B (2012) Thiazolidinediones and PPARgamma agonists: time for a reassessment. Trends Endocrinol Metab 23, 205-215

49. Gray LR, Sultana MR, Rauckhorst AJ et al (2015) Hepatic Mitochondrial Pyruvate Carrier 1 Is Required for Efficient Regulation of Gluconeogenesis and Whole-Body Glucose Homeostasis. Cell Metab 22, 669-681

50. Colca JR, VanderLugt JT, Adams WJ et al (2013) Clinical proof-of-concept study with MSDC-0160, a prototype mTOT-modulating insulin sensitizer. Clin Pharmacol Ther 93, 352-359

51. Kramer W, Müller G and Geisen K (1996) Characterization of the molecular mode of action of the sulfonylurea, glimepiride, at beta-cells. Horm Metab Res 28, 464-468

52. DeFronzo RA and Simonson DC (1984) Oral sulfonylurea agents suppress hepatic glucose production in non-insulin-dependent diabetic individuals. Diabetes Care 7 , $72-80$

53. Taskinen MR, Beltz WF, Harper I et al (1986) Effects of 
NIDDM on very-low-density lipoprotein triglyceride and apolipoprotein B metabolism. Studies before and after sulfonylurea therapy. Diabetes 35, 1268-1277

54. Howard BV, Xiaoren P, Harper I, Foley JE, Cheung MC and Taskinen MR (1985) Effect of sulfonylurea therapy on plasma lipids and high-density lipoprotein composition in non-insulin-dependent diabetes mellitus. Am J Med 79, 78-85

55. Monami M, Vitale V, Ambrosio ML et al (2012) Effects on lipid profile of dipeptidyl peptidase 4 inhibitors, pioglitazone, acarbose, and sulfonylureas: meta-analysis of placebo-controlled trials. Adv Ther 29, 736-746

56. Gerstein HC, Bosch J, Dagenais GR et al (2012) Basal insulin and cardiovascular and other outcomes in dysglycemia. N Engl J Med 367, 319-328

57. Ben-Shlomo S, Zvibel I, Shnell M et al (2011) Glucagonlike peptide-1 reduces hepatic lipogenesis via activation of AMP-activated protein kinase. J Hepatol 54, 12141223

58. Ding $X$, Saxena NK, Lin S, Gupta NA and Anania FA (2006) Exendin-4, a glucagon-like protein-1 (GLP-1) receptor agonist, reverses hepatic steatosis in ob/ob mice. Hepatology 43, 173-181

59. Mells JE, Fu PP, Sharma S et al (2012) Glp-1 analog, liraglutide, ameliorates hepatic steatosis and cardiac hypertrophy in C57BL/6J mice fed a Western diet. Am J Physiol Gastrointest Liver Physiol 302, G225-235

60. Armstrong MJ, Gaunt P, Aithal GP et al (2015) Liraglutide safety and efficacy in patients with non-alcoholic steatohepatitis (LEAN): a multicentre, double-blind, randomised, placebo-controlled phase 2 study. Lancet [Epub ahead of print]

61. Wang XC, Gusdon AM, Liu H and Qu S (2014) Effects of glucagon-like peptide-1 receptor agonists on non-alcoholic fatty liver disease and inflammation. World J Gastroenterol 20, 14821-14830

62. Aroor AR, Habibi J, Ford DA et al (2015) Dipeptidyl peptidase-4 inhibition ameliorates Western diet-induced hepatic steatosis and insulin resistance through hepatic lipid remodeling and modulation of hepatic mitochondrial function. Diabetes 64, 1988-2001

63. Kato H, Nagai Y, Ohta A et al (2015) Effect of sitagliptin on intrahepatic lipid content and body fat in patients with type 2 diabetes. Diabetes Res Clin Pract 109, 199-205

64. Macauley M, Hollingsworth KG, Smith FE et al (2015) Effect of vildagliptin on hepatic steatosis. J Clin Endocrinol Metab 100, 1578-1585

65. Scirica BM, Bhatt DL, Braunwald E et al (2013) Saxagliptin and cardiovascular outcomes in patients with type 2 diabetes mellitus. N Engl J Med 369, 1317-1326

66. Ferrannini E, Muscelli E, Frascerra S et al (2014) Metabolic response to sodium-glucose cotransporter 2 inhibition in type 2 diabetic patients. J Clin Invest 124, 499-508

67. Bolinder J, Ljunggren Ö, Johansson L et al (2014) Dapagliflozin maintains glycaemic control while reducing weight and body fat mass over 2 years in patients with type 2 diabetes mellitus inadequately controlled on metformin. Diabetes Obes Metab 16, 159-169

68. Yokono M, Takasu T, Hayashizaki Y et al (2014) SGLT2 selective inhibitor ipragliflozin reduces body fat mass by increasing fatty acid oxidation in high-fat diet-induced obese rats. Eur J Pharmacol 727, 66-74

69. Neumiller JJ (2014) Empagliflozin: a new sodium-glucose co-transporter 2 (SGLT2) inhibitor for the treatment of type 2 diabetes. Drugs Context 3, 212262

70. Bode B, Stenlöf K, Harris S et al (2015) Long-term efficacy and safety of canagliflozin over 104 weeks in patients aged 55-80 years with type 2 diabetes. Diabetes Obes Metab 17, 294-303

71. Zinman B, Wanner C, Lachin JM et al (2015) Empagliflozin, Cardiovascular Outcomes, and Mortality in Type 2 Diabetes. N Engl J Med 373, 2117-2128

72. Pfefferkorn JA (2013) Strategies for the design of hepatoselective glucokinase activators to treat type 2 diabetes. Expert Opin Drug Discov 8, 319-330

73. Sammons MF and Lee EC (2015) Recent progress in the development of small-molecule glucagon receptor antagonists. Bioorg Med Chem Lett 25, 4057-4064

74. Erion DM, Lapworth A, Amor PA et al (2014) The hepatoselective glucokinase activator PF-04991532 ameliorates hyperglycemia without causing hepatic steatosis in diabetic rats. PLoS One 9, e97139

75. Guan HP, Yang X, Lu K et al (2015) Glucagon receptor antagonism induces increased cholesterol absorption. J Lipid Res 56, 2183-2195

76. Sazonov V, Maccubbin D, Sisk CM and Canner PL (2013) Effects of niacin on the incidence of new onset diabetes and cardiovascular events in patients with normoglycaemia and impaired fasting glucose. Int J Clin Pract 67, 297-302

77. McKenney J (2004) New perspectives on the use of niacin in the treatment of lipid disorders. Arch Intern Med 164, 697-705

78. Capuzzi DM, Morgan JM, Brusco OA Jr and Intenzo CM (2000) Niacin dosing: relationship to benefits and adverse effects. Curr Atheroscler Rep 2, 64-71

79. Drood JM, Zimetbaum PJ and Frishman WH (1991) Nicotinic-Acid for the Treatment of Hyperlipoproteinemia. J Clin Pharmacol 31, 641-650

80. Elam MB, Hunninghake DB, Davis KB et al (2000) Effect of niacin on lipid and lipoprotein levels and glycemic control in patients with diabetes and peripheral arterial disease: the ADMIT study: A randomized trial. Arterial Disease Multiple Intervention Trial. JAMA 284, 12631270

81. Downs JR, Clearfield M, Weis S et al (1998) Primary prevention of acute coronary events with lovastatin in men and women with average cholesterol levels: results of AFCAPS/TexCAPS. Air Force/Texas Coronary Atherosclerosis Prevention Study. JAMA 279, 1615-1622

82. Shepherd J, Cobbe SM, Ford I et al (1995) Prevention of coronary heart disease with pravastatin in men with hypercholesterolemia. West of Scotland Coronary Prevention Study Group. N Engl J Med 333, 1301-1307

83. McFarlane SI, Muniyappa R, Francisco R and Sowers JR (2002) Clinical review 145: Pleiotropic effects of statins: lipid reduction and beyond. J Clin Endocrinol Metab 87, 1451-1458

84. Sugiyama S, Fukushima H, Kugiyama K et al (2007) 
Pravastatin improved glucose metabolism associated with increasing plasma adiponectin in patients with impaired glucose tolerance and coronary artery disease. Atherosclerosis 194, e43-51

85. Preiss D, Seshasai SR, Welsh P et al (2011) Risk of incident diabetes with intensive-dose compared with moderate-dose statin therapy: a meta-analysis. JAMA 305, 2556-2564

86. Sattar N, Preiss D, Murray HM et al (2010) Statins and risk of incident diabetes: a collaborative meta-analysis of randomised statin trials. Lancet 375, 735-742

87. Mullard A (2012) Cholesterol-lowering blockbuster candidates speed into Phase III trials. Nat Rev Drug Discov $11,817-819$

88. Roth EM, Taskinen MR, Ginsberg HN et al (2014) Monotherapy with the PCSK9 inhibitor alirocumab versus ezetimibe in patients with hypercholesterolemia: results of a 24 week, double-blind, randomized Phase 3 trial. Int J Cardiol 176, 55-61

89. Okamoto $\mathrm{H}$, Yonemori $\mathrm{F}$, Wakitani $\mathrm{K}$, Minowa $\mathrm{T}$, Maeda $\mathrm{K}$ and Shinkai H (2000) A cholesteryl ester transfer protein inhibitor attenuates atherosclerosis in rabbits. Nature 406, 203-207

90. de Grooth GJ, Kuivenhoven JA, Stalenhoef AF et al (2002) Efficacy and safety of a novel cholesteryl ester transfer protein inhibitor, JTT-705, in humans - A randomized phase II dose-response study. Circulation 105, 2159-2165

91. Nicholls SJ, Brewer HB, Kastelein JJ et al (2011) Effects of the CETP Inhibitor Evacetrapib Administered as Monotherapy or in Combination With Statins on HDL and LDL Cholesterol A Randomized Controlled Trial. JAMA 306, 2099-2109

92. Barter PJ, Rye KA, Tardif JC et al (2011) Effect of torcetrapib on glucose, insulin, and hemoglobin A1c in subjects in the Investigation of Lipid Level Management to Understand its Impact in Atherosclerotic Events (ILLUMINATE) trial. Circulation 124, 555-562

93. Kharitonenkov A and Shanafelt AB (2009) FGF21: a novel prospect for the treatment of metabolic diseases. Curr Opin Investig Drugs 10, 359-364

94. Lin Z, Tian H, Lam KS et al (2013) Adiponectin mediates the metabolic effects of FGF21 on glucose homeostasis and insulin sensitivity in mice. Cell Metab 17, 779-789

95. Bernardo B, Lu M, Bandyopadhyay G et al (2015) FGF21 does not require interscapular brown adipose tissue and improves liver metabolic profile in animal models of obesity and insulin-resistance. Sci Rep 5, 11382

96. Gaich G, Chien JY, Fu H et al (2013) The effects of LY2405319, an FGF21 analog, in obese human subjects with type 2 diabetes. Cell Metab 18, 333-340

97. Petersen KF, Dufour S, Hariri A et al (2010) Apolipoprotein $\mathrm{C} 3$ gene variants in nonalcoholic fatty liver disease. N Engl J Med 362, 1082-1089

98. Petersen KF, Dufour S, Feng J et al (2006) Increased prevalence of insulin resistance and nonalcoholic fatty liver disease in Asian-Indian men. Proc Natl Acad Sci U S A 103, 18273-18277

99. Lee HY, Birkenfeld AL, Jornayvaz FR et al (2011) Apolipoprotein CIII overexpressing mice are predisposed to diet-induced hepatic steatosis and hepatic insulin resistance. Hepatology 54, 1650-1660

100. Jørgensen $A B$, Frikke-Schmidt $R$, Nordestgaard $B G$ and Tybjærg-Hansen A (2014) Loss-of-function mutations in APOC3 and risk of ischemic vascular disease. $N$ Engl J Med 371, 32-41

101. Crosby J, Peloso GM, Auer PL et al (2014) Loss-of-function mutations in APOC3, triglycerides, and coronary disease. N Engl J Med 371, 22-31

102. Timpson NJ, Walter K, Min JL et al (2014) A rare variant in APOC3 is associated with plasma triglyceride and VLDL levels in Europeans. Nat Commun 5, 4871

103. Gaudet D, Brisson D, Tremblay K et al (2014) Targeting APOC3 in the familial chylomicronemia syndrome. $\mathrm{N}$ Engl J Med 371, 2200-2206

104. Cao J, Zhou Y, Peng H et al (2011) Targeting Acyl-CoA: diacylglycerol acyltransferase 1 (DGAT1) with small molecule inhibitors for the treatment of metabolic diseases. J Biol Chem 286, 41838-41851

105. Chen HC and Farese RV Jr (2005) Inhibition of triglyceride synthesis as a treatment strategy for obesity: lessons from DGAT1-deficient mice. Arterioscler Thromb Vasc Biol 25, 482-486

106. Yamamoto T, Yamaguchi H, Miki H et al (2010) Coenzyme A: diacylglycerol acyltransferase 1 inhibitor ameliorates obesity, liver steatosis, and lipid metabolism abnormality in KKAy mice fed high-fat or high-carbohydrate diets. Eur J Pharmacol 640, 243-249

107. Choi CS1, Savage DB, Kulkarni A et al (2007) Suppression of diacylglycerol acyltransferase-2 (DGAT2), but not DGAT1, with antisense oligonucleotides reverses diet-induced hepatic steatosis and insulin resistance. J Biol Chem 282, 22678-22688

108. Denison H, Nilsson C, Kujacic M et al (2013) Proof of mechanism for the DGAT1 inhibitor AZD7687: results from a first-time-in-human single-dose study. Diabetes Obes Metab 15, 136-143

109. Wierzbicki AS, Hardman TC and Viljoen A (2012) New lipid-lowering drugs: an update. Int J Clin Pract 66, 270280

110. Lee YJ, Ko EH, Kim JE et al (2012) Nuclear receptor PPARgamma-regulated monoacylglycerol O-acyltransferase 1 (MGAT1) expression is responsible for the lipid accumulation in diet-induced hepatic steatosis. Proc Natl Acad Sci U S A 109, 13656-13661

111. Hall AM, Soufi N, Chambers KT et al (2014) Abrogating monoacylglycerol acyltransferase activity in liver improves glucose tolerance and hepatic insulin signaling in obese mice. Diabetes 63, 2284-2296

112. Hall AM, Kou K, Chen Z et al (2012) Evidence for regulated monoacylglycerol acyltransferase expression and activity in human liver. J Lipid Res 53, 990-999 